\title{
Myocardial extracellular volume by T1 mapping: a new marker of arrhythmia in mitral valve prolapse
}

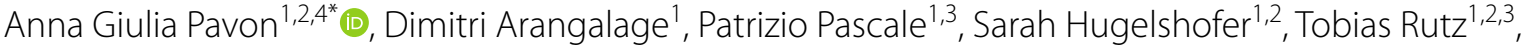 \\ Alessandra Pia Porretta', Mathieu Le Bloa', Olivier Muller ${ }^{1,3}$, Etienne Pruvot ${ }^{1,3}$, Juerg Schwitter ${ }^{1,2,3}$ and \\ Pierre Monney ${ }^{1,2,3}$
}

\begin{abstract}
Objectives: We aimed to evaluate the relationship between mitral annular disjunction (MAD) severity and myocardial interstitial fibrosis at the left ventricular (LV) base in patients with mitral valve prolapse (MVP), and to assess the association between severity of interstitial fibrosis and the occurrence of ventricular arrhythmic events.

Background: In MVP, MAD has been associated with myocardial replacement fibrosis and arrhythmia, but the importance of interstitial fibrosis remains unknown.

Methods: In this retrospective study, 30 patients with MVP and MAD (MVP-MAD) underwent cardiovascular magnetic resonance (CMR) with assessment of MAD length, late gadolinium enhancement (LGE), and basal segments myocardial extracellular volume (ECVsyn). The control group included 14 patients with mitral regurgitation (MR) but no MAD (MR-NoMAD) and 10 patients with normal CMR (NoMR-NoMAD). Fifteen MVP-MAD patients underwent 24 h-Holter monitoring.
\end{abstract}

Results: LGE was observed in 47\% of MVP-MAD patients and was absent in all controls. ECVsyn was higher in MVPMAD $(30 \pm 3 \%$ vs $24 \pm 3 \%$ MR-NoMAD, $p<0.001$ and vs $24 \pm 2 \%$ NoMR-NoMAD, $p<0.001)$, even in MVP-MAD patients without $L G E(29 \pm 3 \%$ vs $24 \pm 3 \%, p<0.001$ and vs $24 \pm 2 \%, p<0.001$, respectively). MAD length correlated with ECVsyn (rho $=0.61, p<0.001$ ), but not with LGE extent. Four patients had history of out-of-hospital cardiac arrest; LGE and ECVsyn were equally performant to identify those high-risk patients, area under the receiver operating characteristic (ROC) curve 0.81 vs $0.83, p=0.84$ ). Among patients with Holter, $87 \%$ had complex ventricular arrhythmia. ECVsyn was above the cut-off value in all while only $53 \%$ had LGE.

Conclusion: Increase in ECVsyn, a marker of interstitial fibrosis, occurs in MVP-MAD even in the absence of LGE, and was correlated with MAD length and increased risk of out-of-hospital cardiac arrest. ECV should be includedin the CMR examination of MVP patients in an effort to better assess fibrous remodelling as it may provide additional value beyond the assessment of LGE in the arrhythmic risk stratification.

Keywords: Mitral valve prolapse, Cardiovascular magnetic resonance, Mitral annular disjunction, Interstitial fibrosis

*Correspondence: Anna-Giulia.Pavon@chuv.ch; annagiulia.pavon@gmail.com 1 Department of Cardiology, Lausanne University Hospital (CHUV), Rue du Bugnon 46, 1011 Lausanne, Switzerland

Full list of author information is available at the end of the article

\section{Introduction}

With a prevalence of about $2 \%$ in the western population, mitral valve prolapse (MVP) is a relatively common condition, associated with a good overall prognosis [1-3]. However, recent findings suggest an association original author(s) and the source, provide a link to the Creative Commons licence, and indicate if changes were made. The images or other third party material in this article are included in the article's Creative Commons licence, unless indicated otherwise in a credit line to the material. If material is not included in the article's Creative Commons licence and your intended use is not permitted by statutory regulation or exceeds the permitted use, you will need to obtain permission directly from the copyright holder. To view a copy of this licence, visit http://creativecommons.org/licenses/by/4.0/. The Creative Commons Public Domain Dedication waiver (http://creativeco mmons.org/publicdomain/zero/1.0/) applies to the data made available in this article, unless otherwise stated in a credit line to the data. 
between MVP and ventricular arrhythmias (VA) as well as an increased incidence of sudden cardiac death (SCD); hence, the terms "arrhythmic" or "malignant" MVP have been coined [4-6]. The nature of this relationship remains only partially deciphered and identifying prognostic factors and mechanisms associated with these events is currently emerging as a challenging task [2, 7 , 8]. Several prognostic factors have been reported in the literature, including negative $\mathrm{T}$ waves, bileaflet prolapse with leaflet redundancy $[1,8]$, polymorphic premature ventricular contraction (PVC) [2], flail mitral valve leaflet, significant mitral regurgitation (MR) [9], and most interestingly left ventricular (LV) fibrosis and mitral annular disjunction (MAD) which seem to be key predictors of VA $[6,8,10-12]$. From a pathophysiological perspective, the excessive mobility of mitral valve leaflets may induce mechanical stretch of the inferobasal wall and of the papillary muscles leading to LV fibrosis. Fibrosis can be detected by late gadolinium enhancement (LGE) using cardiovascular magnetic resonance (CMR) and typically involves the papillary muscles and the LV basal inferior segment in the vicinity of the prolapsing posterior mitral leaflet $[5,10]$.

Myocardial extracellular volume (ECV) quantification by $\mathrm{T} 1$ mapping has emerged as an accurate tool to detect ECV expansion, a quantitative marker of diffuse myocardial fibrosis in ischemic and non-ischemic cardiomyopathies [13, 14]. Most importantly, ECV has been found to be an independent predictor of adverse outcome in patients with significant aortic stenosis [15, 16]. In patients with $M R$, abnormal native $T 1$ mapping indices and shorter post-contrast $\mathrm{T} 1$ times have been reported, but the clinical significance of these observations and their association with an increased risk of SCD or arrhythmia remain unclear [17-20].

Herein, we aimed to evaluate the relationship between MAD severity and myocardial interstitial fibrosis at the LV base quantified by $\mathrm{T} 1$ mapping in patients with bileaflet MVP. In addition, we sought to assess the association between a history of out-of-hospital cardiac arrest (OHCA) and the extent of interstitial myocardial fibrosis, as well as the relation between myocardial fibrosis and the occurrence of VA on holter monitoring in MVP patients.

\section{Methods}

\section{Study population}

From the CMR registry of the Lausanne University Hospital, we retrospectively included patients with a bileaflet MVP and MAD between January 2011 and October 2019. MVP was defined as a systolic excursion of the mitral leaflets $>2 \mathrm{~mm}$ behind the mitral annular plane in the long axis view, i.e. a displacement of $>2 \mathrm{~mm}$ into the left atrium (LA) [21]. MAD was defined as the apparent systolic separation of the mitral leaflet insertion from the ventricular myocardium [10]. As a control group, we included patients with various degrees of MR identified by echocardiography, who underwent CMR during the same period. Patients with myocardial infarction, myocarditis, hypertrophic cardiomyopathy, infiltrative heart disease, more than mild associated valvular heart disease and/or a LV ejection fraction (LVEF) $<50 \%$ were excluded. Three groups were finally constituted: (1) patients with MVP and MAD (MVP-MAD), (2) patients with MR but without MAD (MR-No MAD), and (3) patients with neither MR nor MAD (No MR-No MAD). Clinical characteristics were collected based on institutional medical records. The CMR registry was approved by the local ethics committee CER-VD (Protocol number 2018-00656) and the patients provided written informed consent for their inclusion.

\section{Cardiovascular magnetic resonance protocol}

Electrocardiogram (ECG)-gated CMR imaging was performed using a 1.5T CMR system (MAGNETOM Aera or Sola, Siemens Healthineers, Erlangen-Germany) with a 32-channel phased-array surface receiver coil. Cine images were acquired using a breath-hold balanced steady-state free precession sequence (bSSFP) in long-axis (2-chamber, 3-chamber and 4-chamber) and short-axis views (8 mm slices without gap, 10-15 slices). Breath-hold ECG-triggered phase contrast velocity sequences for ascending aortic flow evaluation were acquired to estimate the aortic forward flow and calculate the regurgitation fraction. For pre-contrast T1 mapping, we used the ECG-triggered modified LookLocker inversion recovery (MOLLI) sequence (using the scheme $3(3) 3(3) 5$ ) on a single short-axis basal LV slice. The basal slice was defined as the slice position of the most basal cine bSSFP slice, where a complete ring of myocardium was visible throughout diastole and systole. Ten minutes after the administration of a $0.2 \mathrm{mmol} /$ $\mathrm{kg}$ intravenous bolus of Gadobutrol (Gadovist, Bayer Healthcare, Berlin, Germany), LGE images were acquired using a 2D breath-hold phase-sensitive segmented inversion-recovery gradient echo pulse sequence in the same orientations as cine images. Inversion-time was individually optimized to null normal myocardium. Post-contrast T1-mapping was acquired following LGE imaging (typically $20 \mathrm{~min}$ after Gadobutrol bolus injection) using a MOLLI sequence (4(1)3(1)2 scheme). As previously reported in literature [17], pre and post-contrast T1 mapping was optimized to improve the precision of the measurement, in particular pre-contrast $\mathrm{T} 1$ mapping was optimized for measuring long T1s of the order of 
$1000 \mathrm{~ms}$ while the post contrast sequence was optimized for short T1s of the order of $200 \mathrm{~ms}$.

\section{Image analysis}

All CMR examinations were analyzed blinded to clinical characteristics and outcome using the Syngo.via software (Siemens Healthiners) to calculate LV volumes, LV mass and LVEF by delineating endocardial and epicardial borders in the stack of short-axis cine images. The presence of myocardial LGE was visually evaluated and its extent was semi-quantitatively reported according to the American Heart Association 17 segments model [22]. In

\section{T1 mapping}

Pre- and post-contrast T1 mapping images were first visually reviewed to assess quality, and myocardial $\mathrm{T} 1$ relaxation times were then measured using the GTVolume software (GyroTools LLC, Zurich, Switzerland). After manual tracing of the endocardial and epicardial borders, the basal slice of the LV was automatically divided into 6 equal segments and the mean T1 relaxation time was calculated for each segment (Fig. 1, Panel B, C). The synthetic (ECVsyn) was calculated without haematocrit sampling as recently validated by Treibel et al. [24] In particular for ECV calculation the following formula was used [25]:

$$
\mathrm{ECV}=(1-\text { haematocrit }) \times \frac{\left(\frac{1}{\text { T1myocardial post }}\right)-\left(\frac{1}{\text { T1myocardial pre }}\right)}{\left(\frac{1}{\text { T1blood post }}\right)-\left(\frac{1}{\text { T1blood pre }}\right)}
$$

the MVP-MAD group, the MR volume was calculated according to the standard indirect method, by subtracting the forward aortic flow volume from the total LV stroke volume (LV stroke volume - forward aortic flow). The MR fraction was quantified using the following equation: (regurgitant volume $\times 100) /($ LV stroke volume). MR volume and regurgitant fraction corrected for the severity of the prolapse were additionally calculated as previously reported [23]. The MAD length was measured on a 3-chamber view from the LA wall-posterior mitral leaflet junction to the top of the basal LV inferolateral wall during systole (Fig. 1, Panel A).

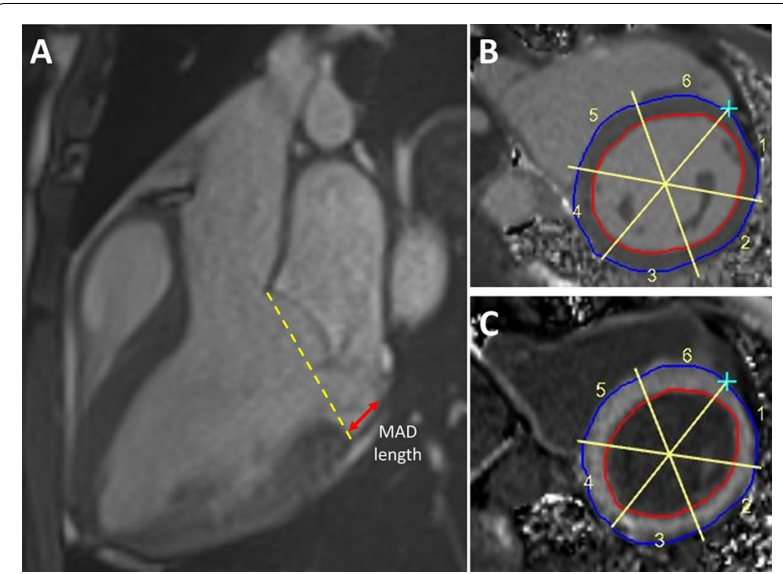

Fig. 1 A Cardiovascular magnetic resonance (CMR) 3 chambers cine image at end-systole for measurement of mitral annular disjunction (MAD) length. B, C Semi-automatic measurement of segmental pre-contrast (B) and post-contrast (C) T1 relaxation time of the basal left ventricular myocardium
According to the literature, synthetic haematocrit was previously validated against conventional haematocrit. For Siemens Aera, a local calibration of the synthetic haematocrit was performed on a population of 20 healthy subjects and more than 800 patients. Following the change in scanner during study period, the local calibration of the synthetic haematocrit was repeated for Siemens Sola on a population of 256 patients. In both cases, a linear regression analysis was used to create a formula allowing synthetic haematocrit estimation from the measured blood relaxivity (R1) (Additional file 1: Figure S1). ECVsyn was then calculated using synthetic haematocrit instead of measured haematocrit in the formula.

ECVsyn expansion was considered to be significant with an ECVsyn $>27 \%$. The reproducibility of $\mathrm{T} 1$ relaxation time and ECVsyn measurement was assessed on a random sample of 15 patients, analyzed by two different operators, blinded to clinical data and both experienced in CMR examination analysis.

\section{Objectives and endpoints definitions}

The main objective of the study was to compare the severity of myocardial fibrosis (extent of LGE, pre-contrast $\mathrm{T} 1$ relaxation time and ECVsyn) at the basal LV myocardium and the length of MAD in MVP patients with and without a recent history of unexplained resuscitated OHCA. As a secondary objective, we assessed the relation between the severity of myocardial fibrosis and the incidence of PVC and of complex PVC (grade 4 or 5 according to Lown classification) in a subgroup of 15 patients with suspected arrhythmic MVP, who performed a $24 \mathrm{~h}$ Holter monitoring. 


\section{Statistical analysis}

Continuous variables were expressed as mean $\pm \mathrm{SD}$ or median [interquartile range] where appropriate, and categorical variables as numbers and percentages (n, \%) respectively. Gaussian distribution was determined with the skewness and kurtosis test for normality. Differences in continuous variables between two groups were analysed with the independent-sample Student's $t$ test in case of normal distribution or the Mann-Whitney test in case of non-normal distribution. Comparisons between three groups were performed by ANOVA with post-hoc Bonferroni correction. Comparison of categorical variables was performed with the $x^{2}$ test. Correlation analysis was performed using Spearman's correlation. Receiver operating characteristic (ROC) analysis was used to determine the optimal cut-off value of selected CMR parameters (LGE and ECVsyn) associated with arrhythmic endpoints. Considering the inhomogeneous distribution of pre-contrast T1 relaxation times and ECVsyn, with the highest values typically located in the inferior and inferolateral region (i.e. at the close vicinity of the posterior mitral leaflet attachment), the average values of the basal inferior and inferolateral segments were used for association analyses with arrhythmic endpoints.

Reproducibility analyses for the measurement of myocardial pre-contrast $\mathrm{T} 1$ and ECVsyn were performed using Pearson's correlation and Bland-Altman statistics.
All statistical analyses were performed using STATA 16.0 (StataCorp, College Station, Texas, USA). A $\mathrm{p}<0.05$ was considered statistically significant.

\section{Results}

A total of 38 patients with MVP and MAD were identified. Five patients with complex congenital heart disease and 3 patients who already underwent mitral valve repair were excluded leaving 30 patients in the MVP-MAD group. Thirty-eight patients with various degrees of MR detected by echocardiography and without MAD where selected as controls. Four patients were excluded because of poor image quality, 6 patients because of previous myocardial infarction and 4 patients because of myocarditis. Among the remaining 24 patients, 14 had mild to severe MR (MR-no MAD) and 10 had no or only a trace MR (no MR-no MAD).

\section{Baseline clinical and morphological characteristics}

Mean age was $50 \pm 19$ years and similar across the three groups $(\mathrm{p}=0.82)$, and $43 \%$ were females. LV end-diastolic volume index was higher in the MVP-MAD group than in the MR-No MAD and the No MR-No MAD groups $\left(102 \pm 29 \mathrm{ml} / \mathrm{m}^{2}\right.$ vs $81 \pm 17 \mathrm{ml} / \mathrm{m}^{2}, \mathrm{p}=0.018$, and vs $82 \pm 16 \mathrm{ml} / \mathrm{m}^{2}, \mathrm{p}=0.048$ respectively), but there was similar LVEF (Table 1). In the MR-No MAD group, MR severity grade assessed by echocardiography was mild

Table 1 Baseline clinical and cardiovascular magnetic resonance (CMR) characteristics

\begin{tabular}{|c|c|c|c|c|c|}
\hline & \multirow{2}{*}{$\begin{array}{l}\text { MVP-MAD } \\
n=30\end{array}$} & \multicolumn{4}{|c|}{ Control group (No MVP) } \\
\hline & & $\begin{array}{l}\text { MR-NoMAD } \\
n=14\end{array}$ & Pvs MVP-MAD & $\begin{array}{l}\text { NoMR-NoMAD } \\
n=10\end{array}$ & Pvs MVP-MAD \\
\hline Age (y) & $50 \pm 17$ & $53 \pm 22$ & 0.70 & $46 \pm 21$ & 0.53 \\
\hline Male, n (\%) & $18(60 \%)$ & $7(50 \%)$ & 0.75 & $6(60 \%)$ & 1.0 \\
\hline Weight (kg) & $72 \pm 12$ & $70 \pm 14$ & 0.69 & $82 \pm 13$ & 0.02 \\
\hline Height (cm) & $177 \pm 10$ & $170 \pm 10$ & 0.04 & $179 \pm 11$ & 0.65 \\
\hline Hypertension, n (\%) & $2(7 \%)$ & $6(43 \%)$ & 0.008 & $2(20 \%)$ & 0.26 \\
\hline Active smoker, n (\%) & $3(10 \%)$ & $1(7 \%)$ & 1.0 & $1(10 \%)$ & 1.0 \\
\hline Hyperlipidaemia, n (\%) & $2(7 \%)$ & $2(14 \%)$ & 0.58 & $2(20 \%)$ & 0.26 \\
\hline LVEDV (ml) & $192 \pm 56$ & $148 \pm 39$ & 0.01 & $164 \pm 29$ & 0.14 \\
\hline LVEDVI $\left(\mathrm{ml} / \mathrm{m}^{2}\right)$ & $102 \pm 29$ & $81 \pm 17$ & 0.018 & $82 \pm 16$ & 0.048 \\
\hline LVESV (ml) & $84 \pm 32$ & $60 \pm 21$ & 0.02 & $70 \pm 12$ & 0.20 \\
\hline LVEF (\%) & $57 \pm 8$ & $60 \pm 8$ & 0.32 & $57 \pm 3$ & 0.99 \\
\hline LV mass index $\left(\mathrm{g} / \mathrm{m}^{2}\right)$ & $66 \pm 16$ & $62 \pm 15$ & 0.45 & $63 \pm 9$ & 0.59 \\
\hline MAD length (mm) & $9.4[7.1-12.3]$ & - & & - & \\
\hline Presence of LGE, n (\%) & $14(47 \%)$ & $0(0 \%)$ & 0.002 & $0(0 \%)$ & 0.007 \\
\hline N of positive segments $(n=14)$ & $3[3-4]$ & 0 & & 0 & \\
\hline Basal slice pre-Gd T1 (ms) & $1067 \pm 45$ & $1029 \pm 37$ & 0.009 & $1029 \pm 26$ & 0.016 \\
\hline Basal slice ECVsyn (\%) & $30 \pm 3$ & $24 \pm 3$ & $<0.0001$ & $24 \pm 2$ & $<0.0001$ \\
\hline
\end{tabular}

ECVsyn synthetic extracellular volume fraction, $L V$ left ventricle, LVEDV left ventricle end-diastolic volume, LVEDVI left ventricle end-diastolic volume index, LVEF left ventricle ejection fraction, $L G E$ late gadolinium enhancement $G d$ gadolinium, MAD mitral annular disjunction, MVP mitral valve prolapse 
in 7 patients $(50 \%)$, moderate in $5(36 \%)$ and severe in 2 (14\%).

In MVP-MAD the maximum MAD length was $9.4 \mathrm{~mm}$ [7.1-12.3]. Phase contrast aortic flow data were available in only 22 MVP-MAD patients and the mean mitral regurgitant fraction was $31 \pm 17 \%$. After correction for the prolapsing volume, the mean MR fraction was $16 \pm 21 \%$. MR severity grade assessed by echocardiography was available in $27 \mathrm{MVP}-\mathrm{MAD}$ patients and MR

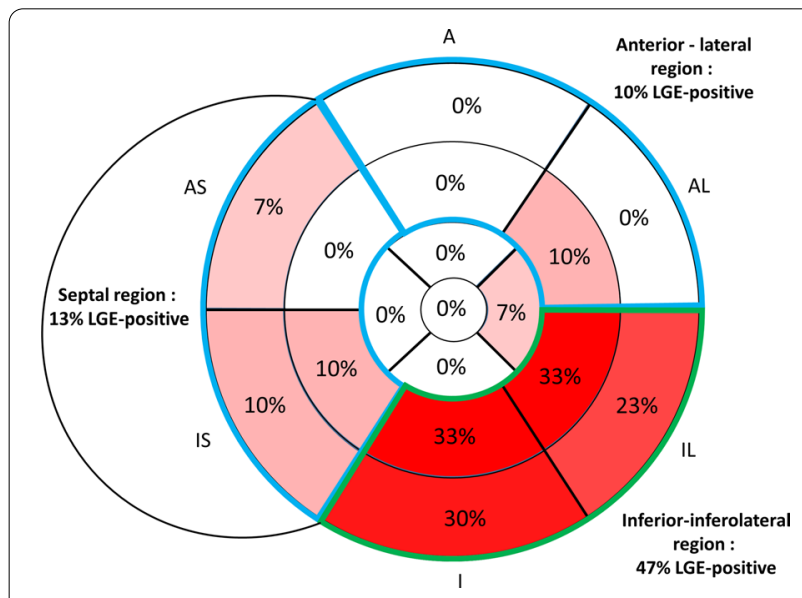

Fig. 2 Distribution of late gadolinium enhancement (LGE) positivity in patients with mitral valve prolapse (MVP) and MAD $(n=30) . A$ anterior, AL anterolateral, IL inferolateral, / inferior, IS inferoseptal, AS anteroseptal was graded as mild in 9 (50\%), moderate in $14(36 \%)$ and severe in $4(14 \%)$ patients.

\section{Tissue characterization}

LGE was present in 14 MVP-MAD patients (47\%) and was absent in the two other groups. In LGE-positive patients, the median LGE extent was 3 [3-4] segments, and LGE was most frequently detected in the basal to mid-ventricular inferior and inferolateral walls (i.e. in the vicinity of the posterior mitral leaflet attachments) compared with the anterior and antero-lateral walls $(47 \%$ vs $10 \%, \mathrm{p}=0.002)$ and the antero-septal and infero-septal walls (47\% vs $13 \%, \mathrm{p}=0.005$ ) (Fig. 2).

Native T1 relaxation was higher in MVP-MAD in comparison with MR-No MAD $(1067 \pm 45 \mathrm{~ms}$ vs $1029 \pm 37 \mathrm{~ms}, \mathrm{p}=0.009)$ and No MR-No MAD $(1029 \pm 26 \mathrm{~ms}, \mathrm{p}=0.016)$ (Fig. 3). LGE negative MVPMAD patients had a native T1 similar to LGE positive MVP-MAD patients $(1057 \pm 49 \mathrm{~ms}$ vs $1078 \pm 39 \mathrm{~ms}$, $\mathrm{p}=0.19$ ), and significantly higher than controls $(1057 \pm 49 \mathrm{~ms}$ vs $1029 \pm 32 \mathrm{~ms}, \mathrm{p}=0.04)$.

The ECVsyn was higher in MVP-MAD compared with MR-No MAD $(30 \pm 3 \%$ vs $24 \pm 3 \%, \mathrm{p}<0.001)$ and No MR-No MAD ( $30 \pm 3 \%$ vs $24 \pm 2 \%, \mathrm{p}<0.001)$ (Fig. 4). In the MVP-MAD group, LGE negative patients had ECVsyn values similar to LGE positive patients $(29 \pm 3 \%$ vs $31 \pm 3 \%, p=0.19)$, and significantly higher than controls $(29 \pm 3 \%$ vs $24 \pm 2 \%, \mathrm{p}<0.001)$. Interestingly, $87 \%$ of the MVP-MAD patients had an

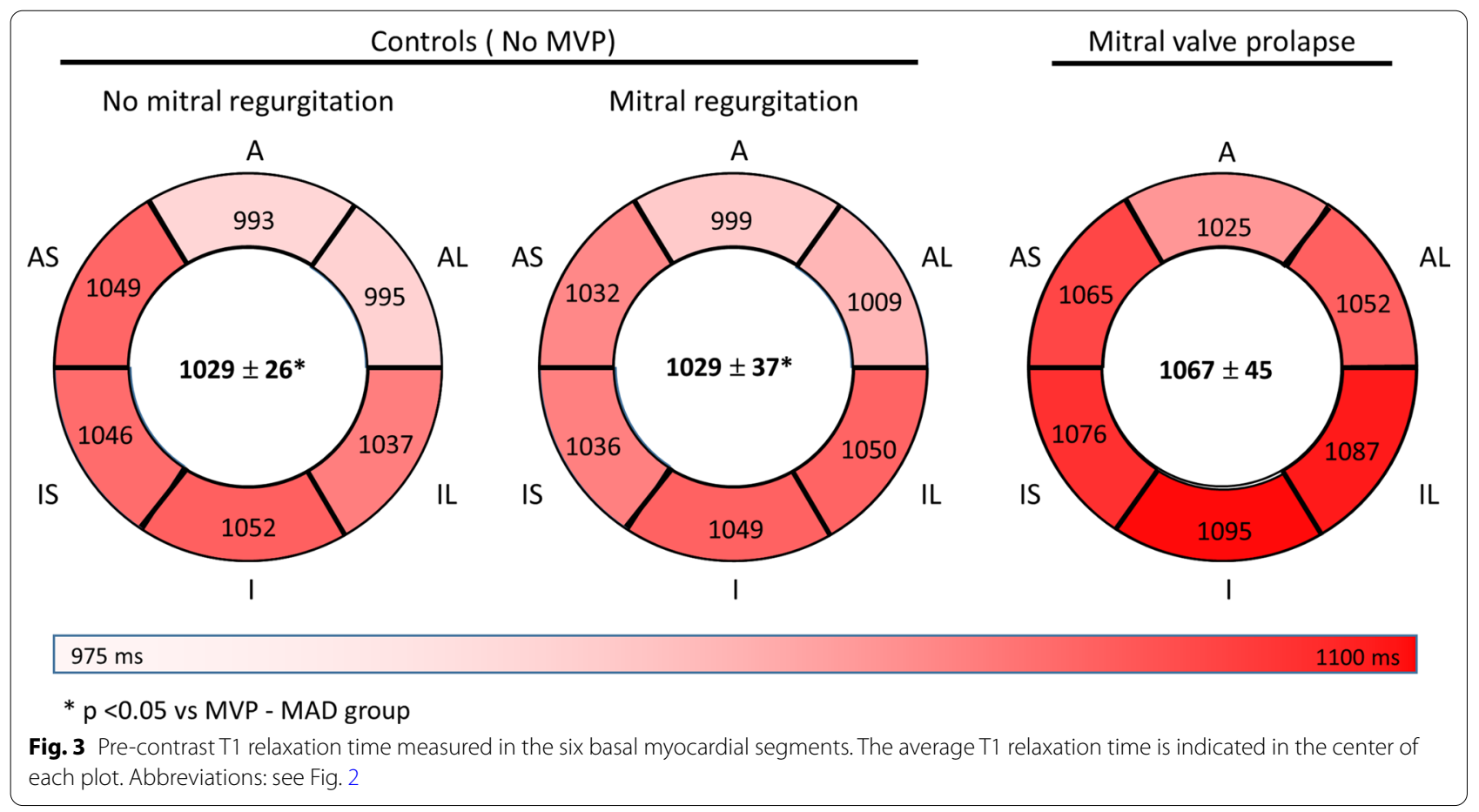




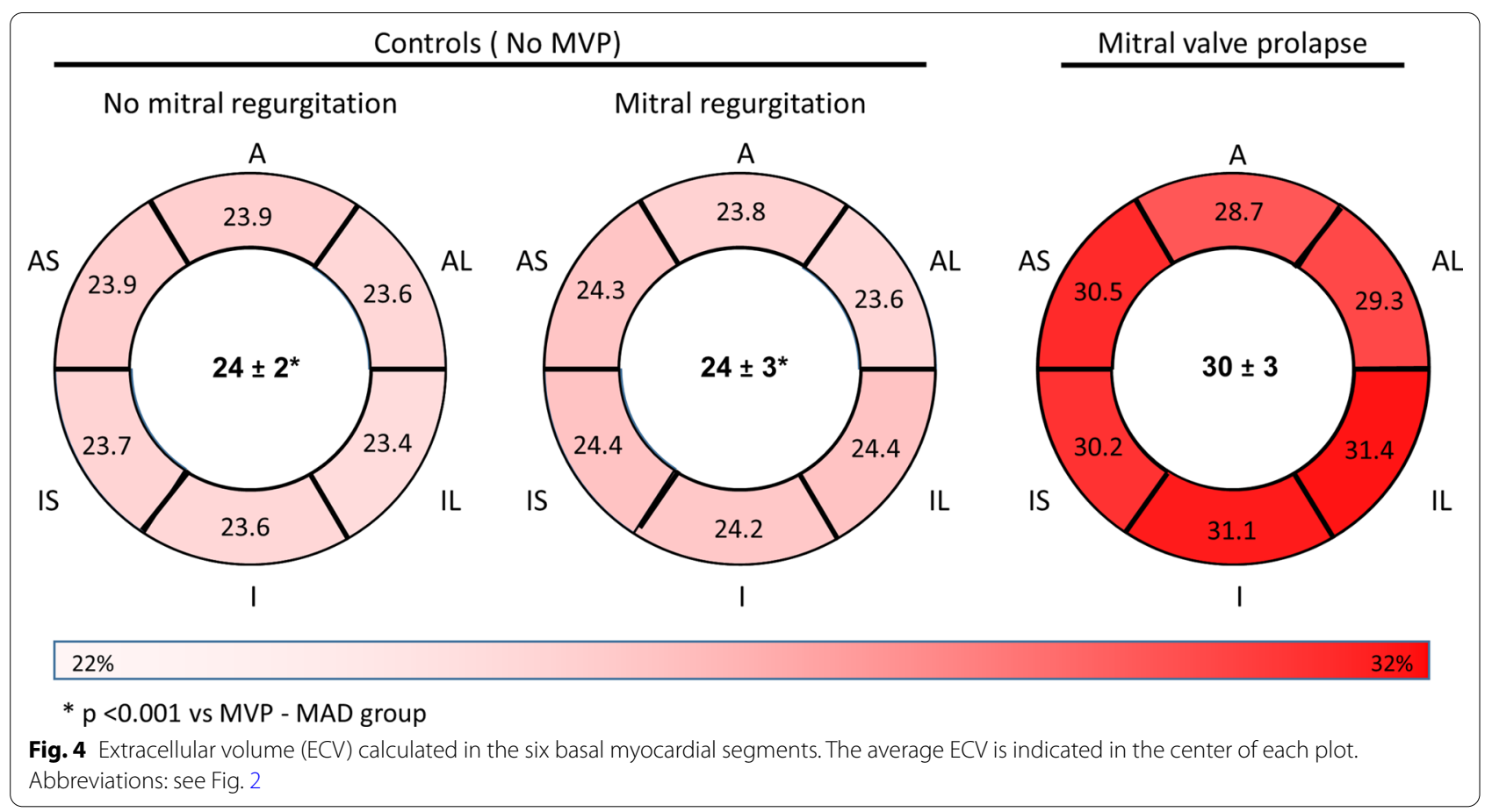

ECVsyn above the upper normal limit (>27\%). This proportion was $93 \%$ in LGE-positive vs $81 \%$ in LGEnegative patients $(\mathrm{p}=0.35)$, and no significant correlation was found between LGE extent and ECVsyn (rho $=0.10, \mathrm{p}=0.60$ ).

No significant correlation was found between MAD length and LGE extent (rho $=-0.01, \mathrm{p}=0.97$ ) or between MAD length and pre-contrast native $\mathrm{T} 1$ (rho $=0.26, \mathrm{p}=0.16$ ) but a moderate to high correlation was found between MAD length and ECVsyn $(r=0.61$, $\mathrm{p}<0.001$ ) (Fig. 5).

Among the 27 MVP-MAD patients with echocardiographic quantification of MR severity, no significant difference was observed among the three MR severity grades for MAD length (8.4 [7.1-10.2] with mild MR vs 10.9 [9-14.6] with moderate MR vs 8.1 [4.2-12.4] $\mathrm{mm}$ with severe MR, respectively, $\mathrm{p}=0.16)$, LGE extent $(0$ [0-2] vs 0 [0-4] vs 3 [1.5-3.5] segments, $\mathrm{p}=0.58)$, T1 relaxation time $(1075 \pm 45$ vs $1059 \pm 44$ vs $1042 \pm 37 \mathrm{~ms}$,

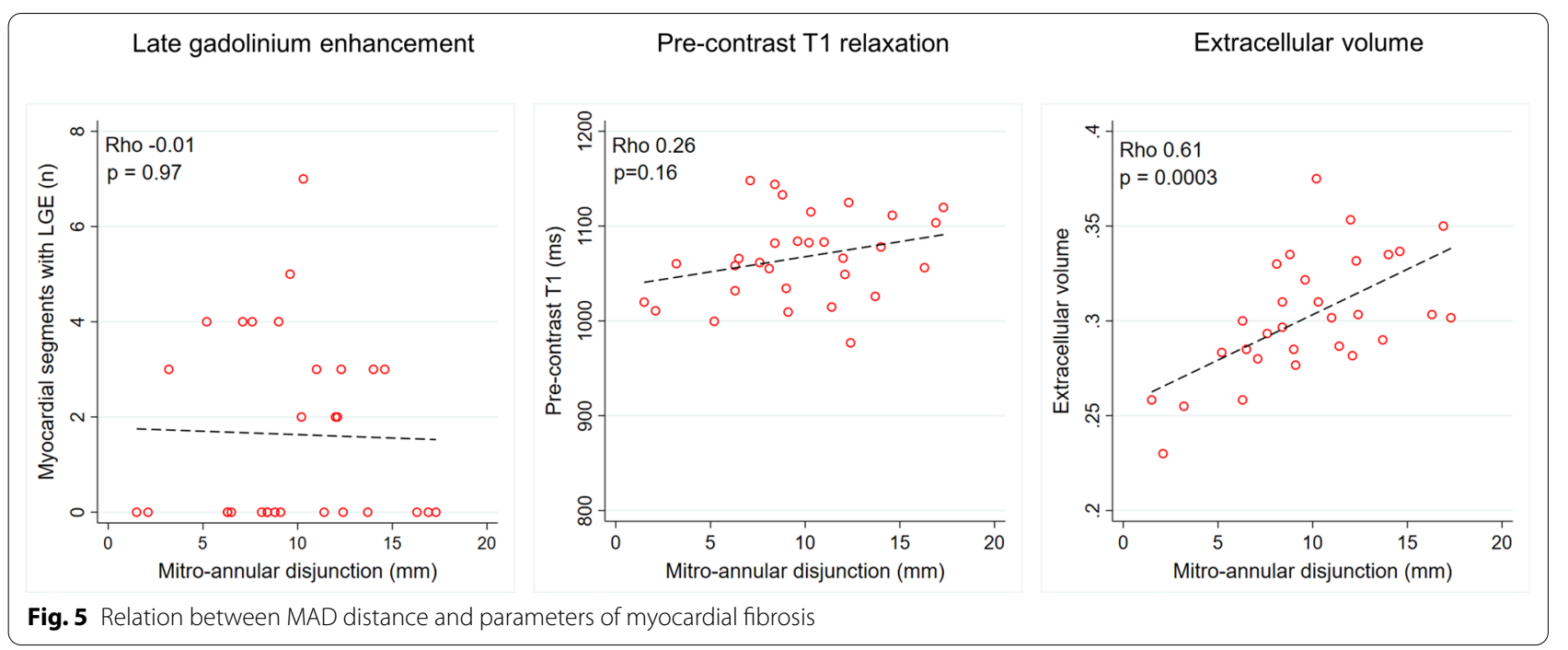


Table 2 Characteristics of the MVP-MAD patients with and without history of OHCA

\begin{tabular}{|c|c|c|c|}
\hline & OHCA $(n=4)$ & No OHCA $(n=26)$ & $\mathbf{p}$ \\
\hline Age (y) & $58 \pm 7$ & $49 \pm 18$ & 0.33 \\
\hline Male, n (\%) & $1(25 \%)$ & $17(65 \%)$ & 0.27 \\
\hline Weight (kg) & $85 \pm 9$ & $70 \pm 11$ & 0.01 \\
\hline Height (cm) & $178 \pm 16$ & $177 \pm 10$ & 0.87 \\
\hline LVEDV (ml) & $154 \pm 16$ & $198 \pm 58$ & 0.15 \\
\hline LVEDVI $\left(\mathrm{ml} / \mathrm{m}^{2}\right)$ & $77 \pm 15$ & $106 \pm 29$ & 0.06 \\
\hline LVESV (ml) & $62 \pm 27$ & $87 \pm 32$ & 0.15 \\
\hline LVEF (\%) & $60 \pm 14$ & $56 \pm 8$ & 0.40 \\
\hline LV mass index ( $\left.\mathrm{g} / \mathrm{m}^{2}\right)$ & $60 \pm 6$ & $67 \pm 17$ & 0.43 \\
\hline MAD length (mm) & $11.2[8.7-13.0]$ & $9.1[6.5-12.3]$ & 0.50 \\
\hline Presence of LGE, n (\%) & $4(100 \%)$ & $10(38 \%)$ & 0.04 \\
\hline $\mathrm{N}$ of positive segments & $3.5[2.5-5.5]$ & $0[0-3]$ & 0.02 \\
\hline Basal slice pre-Gd T1 (ms) ${ }^{\mathrm{a}}$ & $1133 \pm 72$ & $1085 \pm 57$ & 0.14 \\
\hline Basal slice ECVsyn (\%) ${ }^{\mathrm{a}}$ & $35 \pm 4$ & $31 \pm 4$ & 0.03 \\
\hline
\end{tabular}

Statistically significant correlations are given in bold

$E C V$ syn synthetic extracellular volume, $L V$ left ventricle, $L V E D V$ left ventricle end-diastolic volume, LVEDVI left ventricle end-diastolic volume index, LVEF left ventricle ejection fraction, LVESV, left ventricular end-systolic volume. LGE late gadolinium enhancement, $G d$ gadolinium, $M A D$ mitral annular disjunction, OHCA out-of-hospital cardiac arrest

${ }^{a}$ Mean value of inferior and inferolateral segments

$\mathrm{p}=0.50)$ and ECVsyn ( $31 \pm 5$ vs $30 \pm 2$ vs $28 \pm 2 \%$, $\mathrm{p}=0.31$.

\section{Associations between, MAD length, myocardial fibrosis, and OHCA}

Among the 30 MVP-MAD patients, 4 (13\%) were referred for evaluation following OHCA. None of the patients had evidence of coronary heart disease on coronary angiogram. The clinical characteristics of the 4 patients with history of OHCA are presented in Table 2. Although there was no difference in MAD length (11.2 [8.7-13.0] vs 9.1 [6.5-12.3] $\mathrm{mm}, \mathrm{p}=0.50$ ), significant differences were found in terms of body weight $(85 \pm 9 \mathrm{~kg}$ in OHCA patients vs $70 \pm 11 \mathrm{~kg}, \mathrm{p}=0.01)$ and presence of LGE (100\% in OHCA patients vs $38 \%, \mathrm{p}=0.04)$. OHCA patients had a higher LGE extent (3.5 [2.5-5.5] vs 0 [0-3] segments positive for $\mathrm{LGE}, \mathrm{p}=0.02)$. No difference was found in terms of pre-contrast T1 values $(1133 \pm 72$ vs $1085 \pm 57 \mathrm{~ms}, \mathrm{p}=0.14$ ) but a significantly higher ECVsyn was measured in OHCA patients $(35.1 \pm 4.4 \%$ vs $30.7 \pm 3.6 \%, p=0.03$ ) (Fig. 6). Regarding the identification of patients with history of OHCA, the presence of LGE achieved a sensitivity of $100 \%$, a specificity of $62 \%$, a positive predictive value of $29 \%$, and a negative predictive value of $100 \%$. For an ECVsyn threshold $>33.5 \%$, they were $75 \%, 85 \%, 43 \%$, and $96 \%$, respectively. The ROC analysis revealed comparable accuracy of ECVsyn and LGE for the detection of patients with previous OHCA (area under the ROC curve 0.832 vs $0.808, \mathrm{p}=0.84$ ).

Of note, MR was mild in 3 and moderate in one OHCA patient. The mean MR severity grade in OHCA tended to be lower than in patients with no previous OHCA $(1.25 \pm 0.51 .91 \pm 0.67, \mathrm{p}=0.07)$, but the difference was not significant.

\section{Association between myocardial fibrosis and arrhythmia}

The baseline characteristics of the 15 patients investigated with a Holter monitoring are presented in Table 3. The median time between Holter monitoring and CMR was 36 days [1-113 days]. The only significant difference between patients with rhythm assessment compared to patients without was a higher ECVsyn of the basal inferoposterior region ( $33 \pm 5$ vs $30 \pm 3 \%, \mathrm{p}=0.03)$. All patients

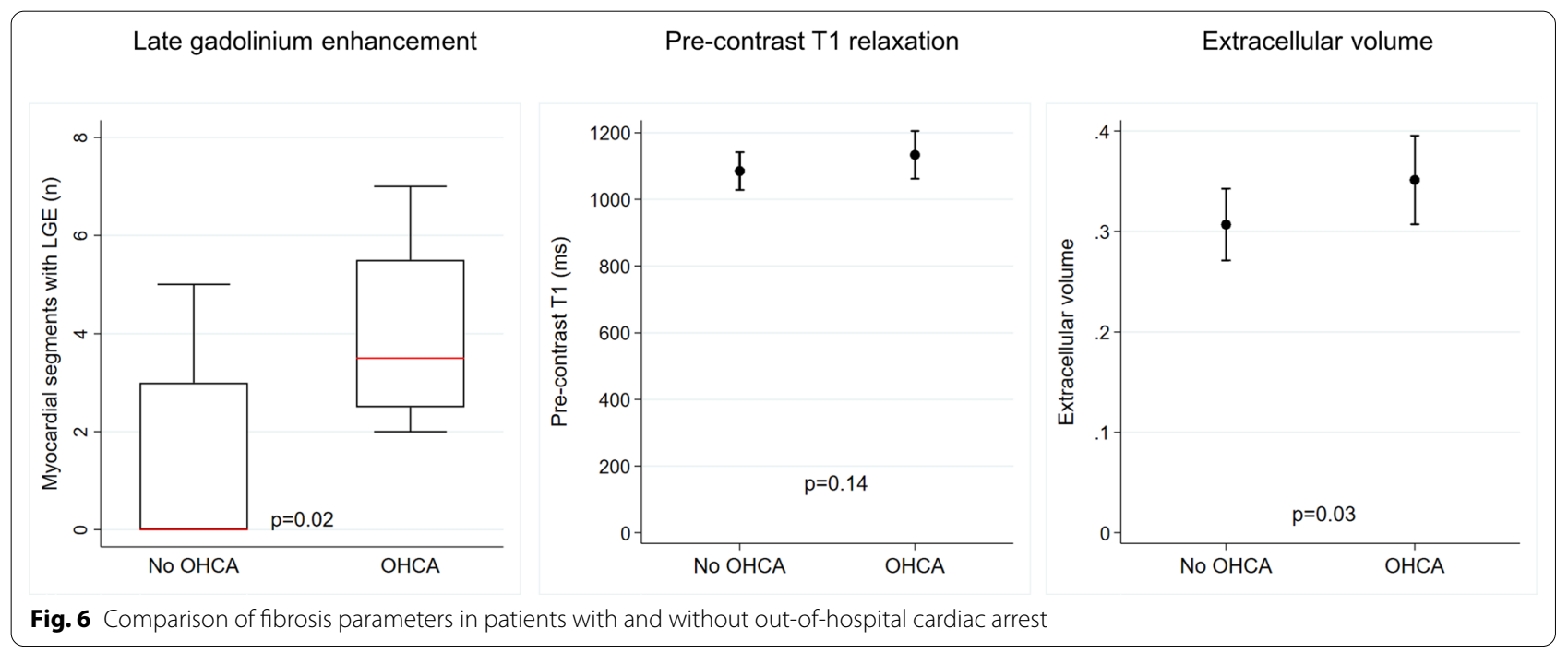


Table 3 Characteristics of patients with and without Holter monitoring

\begin{tabular}{llll}
\hline & Holter $(\mathbf{n}=\mathbf{1 5})$ & No Holter $(\mathbf{n}=\mathbf{1 5})$ & $\mathbf{p}$ \\
\hline Age $(\mathrm{y})$ & $47 \pm 14$ & $53 \pm 19$ & 0.34 \\
Male, $\mathrm{n}(\%)$ & $8(53 \%)$ & $10(67 \%)$ & 0.71 \\
Weight $(\mathrm{kg})$ & $74 \pm 11$ & $70 \pm 13$ & 0.41 \\
Height (cm) & $176 \pm 7$ & $178 \pm 13$ & 0.45 \\
LVEDV (ml) & $191 \pm 45$ & $193 \pm 67$ & 0.93 \\
LVEDVI (ml/m²) & $102 \pm 25$ & $103 \pm 34$ & 0.92 \\
LVESV (ml) & $85 \pm 23$ & $83 \pm 39$ & 0.92 \\
LVEF (\%) & $55 \pm 8$ & $58 \pm 9$ & 0.33 \\
LV mass index (g/m²) & $65 \pm 10$ & $66 \pm 20$ & 0.83 \\
MAD length (mm) & $9.6[7.6-14.6]$ & $9.1[6.3-11.4]$ & 0.30 \\
Presence of LGE, $\mathrm{n}(\%)$ & $8(53 \%)$ & $6(40 \%)$ & 0.72 \\
N of positive segments & $3[2.5-4]$ & $3.5[3-4]$ & 0.59 \\
Basal slice pre-Gd T1 (ms) & $1109 \pm 49$ & $1073 \pm 66$ & 0.11 \\
Basal slice ECVsyn (\%) & $33 \pm 5$ & $30 \pm 3$ & $\mathbf{0 . 0 3}$ \\
\hline
\end{tabular}

Statistically significant correlation is given in bold

ECVsyn synthetic extracellular volume, $L V$ left ventricle, LVEDV left ventricle end-diastolic volume, LVEDVI left ventricle end-diastolic volume index, LVEF left ventricle ejection fraction; $L G E$ late gadolinium enhancement, $G d$ gadolinium, $M A D$ mitral annular disjunction

${ }^{\mathrm{a}}$ Mean value of inferior and inferolateral segments

with Holter experienced PVC with a median incidence of 2.8\% [1.1-10.3\%] of all heartbeats. Complex PVC were detected in 13 patients (87\%) and NSVT (non-sustained ventricular tachycardia) in $10(67 \%)$ with a median of 5 [0-17] runs/24 h. LGE was detected in only 8/15 (53\%) patients while all patients had an ECVsyn $>27 \%$ in the basal infero-posterior region. ECVsyn was not significantly different between patients with and without LGE detected $(34.5 \pm 5$ vs $32 \pm 4 \%, \mathrm{p}=0.47)$. There was no significant difference in PVC count between patients with and without LGE (4.2\% [1.6-8.7\%] vs $2.8 \%$ [0-14.9\%], $\mathrm{p}=0.82)$. Similarly, PVC count was not significantly higher in patients with a higher pre-contrast $\mathrm{T} 1$ ( $\mathrm{T} 1 \geq 1115 \mathrm{~ms}$ corresponding to the median $\mathrm{T} 1$ value of the basal infero-posterior region: $7.1 \%$ [1.3-14.9\%] vs $2.2 \%[0.8-7.1 \%], \mathrm{p}=0.22)$, and in patients with a higher ECVsyn (ECVsyn $\geq 32.0 \%$ corresponding to the median ECVsyn value of the basal infero-posterior region: $2.8 \%$ [1.1-10.3\%] vs $4.2 \%$ [1.2-11.7\%], $\mathrm{p}=0.77$ ).

\section{Intra- and inter-observer reproducibility of T1- and ECVsyn} measurements

Intra-observer $\quad(\mathrm{r}=0.61, \mathrm{p}=0.004 / \mathrm{Mean}$ difference $9 \mathrm{~ms}-95 \%$ levels of agreement-70 to $89 \mathrm{~ms}$ ) and inter-observer $(\mathrm{r}=0.93, \quad \mathrm{p}<0.001 /$ Mean difference $11 \mathrm{~ms}-95 \%$ levels of agreement-25 to $46 \mathrm{~ms}$ ) reproducibility for pre-contrast T1 relaxation time was good. Both intra-observer $(r=0.99, \mathrm{p}<0.001 /$ Mean difference
$0-95 \%$ levels of agreement $-0.3 \%$ to $0.4 \% \mathrm{~ms}$ ) and inter-observer $(\mathrm{r}=0.99, \mathrm{p}<0.001 /$ Mean difference $0 \%$ $\mathrm{ms}-95 \%$ levels of agreement $-0.4 \%$ to $0.4 \%$ ) reproducibility were excellent for ECVsyn calculation (Figs. 7 and 8).

\section{Discussion}

In this retrospective study we found that (1) MVP-MAD patients had a higher ECVsyn indicative of a higher amount of interstitial fibrosis even in the absence of detectable LGE, (2) MAD length was best associated with a higher ECVsyn but not with a higher native T1 or LGE extent, and (3) the presence of LGE and a basal posterior ECVsyn $>33.5 \%$ identified MVP-MAD patients with history of OHCA with similar accuracy.

MVP is a relatively frequent clinical condition with a good overall prognosis. However, VA and SCD may occur with an incidence of $0.2 \%$ to $0.4 \%$ per year in this population $[1,3,9,26]$. Therefore, the identification of risk factors of VA is of paramount importance. Myocardial fibrosis detected by LGE has been associated with the occurrence of VA in several ischemic and non-ischemic cardiac conditions using CMR [27-30]. However, the precise detection of fibrosis may be challenging in areas where the myocardium is not completely replaced by fibrotic tissue [31] as these inhomogeneous areas are characterized by an intermediate intensity (grey zone) due to the intermingling of both viable myocardium and interstitial fibrosis. They are typically present in the border zone of ischemic scars, or associated with non-ischemic cardiomyopathies or inflammatory heart diseases and may promote re-entry pathways for VA [3234]. The newer T1 mapping technique provides complementary information on fibrotic remodelling as it allows a quantitative assessment of myocardial ECV, a marker of extracellular matrix expansion [14, 35, 36]. Although increased ECV identifies patients at higher risk of mortality or heart failure [37], its value in predicting adverse arrhythmic outcomes remains unknown.

In patients with MVP and MAD, increasing evidence supports an association between prolapse severity, curling motion of the basal posterior myocardium and structural changes of the myocardium. The continuous mechanical stretch applied by the MVP has been suggested to induce a fibrotic remodelling of the basal inferior LV wall and the papillary muscles [10, 38], where LGE could be detected in $73 \%$ and $83 \%$ of cases, respectively [10]. MAD itself appears to play an important role in the pathogenesis of VA. In a large cohort of 116 patients with MAD, in which $12 \%$ of the population presented with severe arrhythmic events, Dejgaard et al. observed that MAD was a distinct entity linked to arrhythmia severity, whereas isolated MVP was not [11]. 
Pre-contrast $\mathrm{T} 1$ relaxation
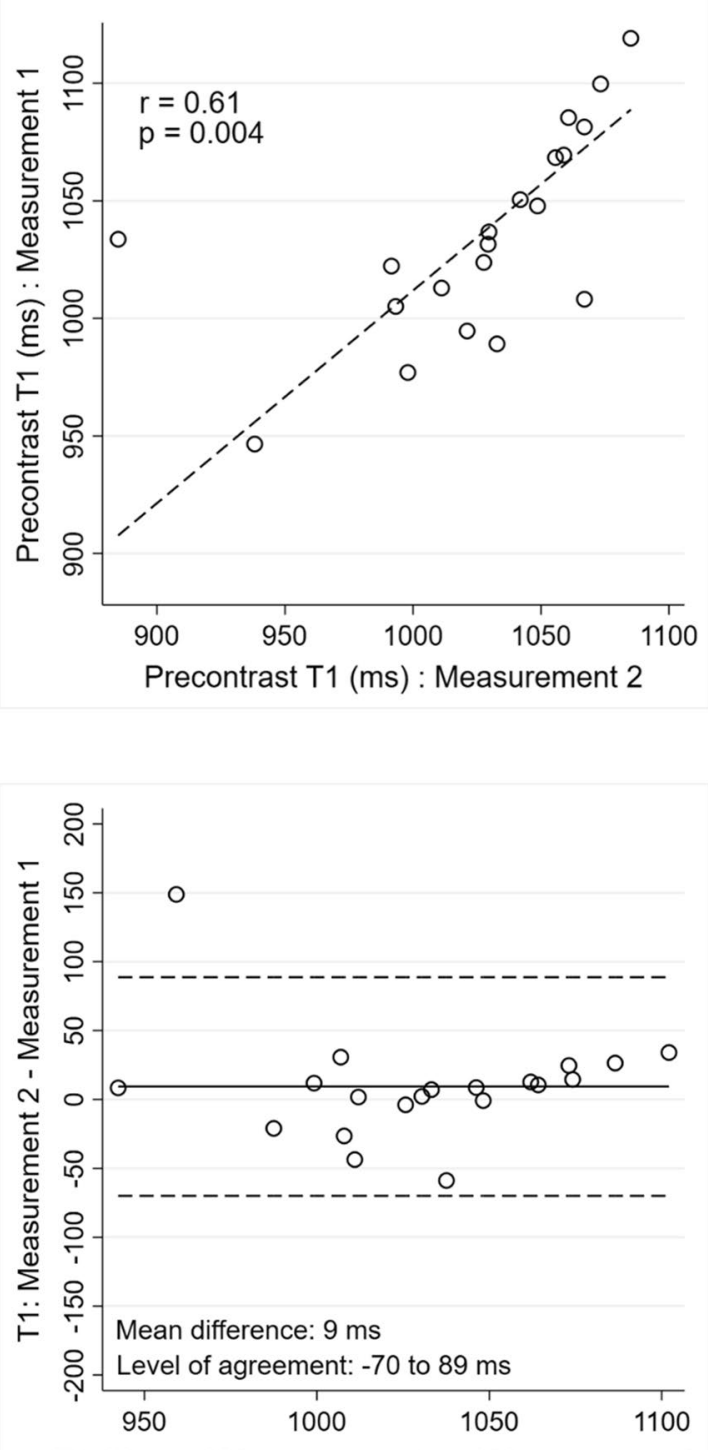

Extracellular volume
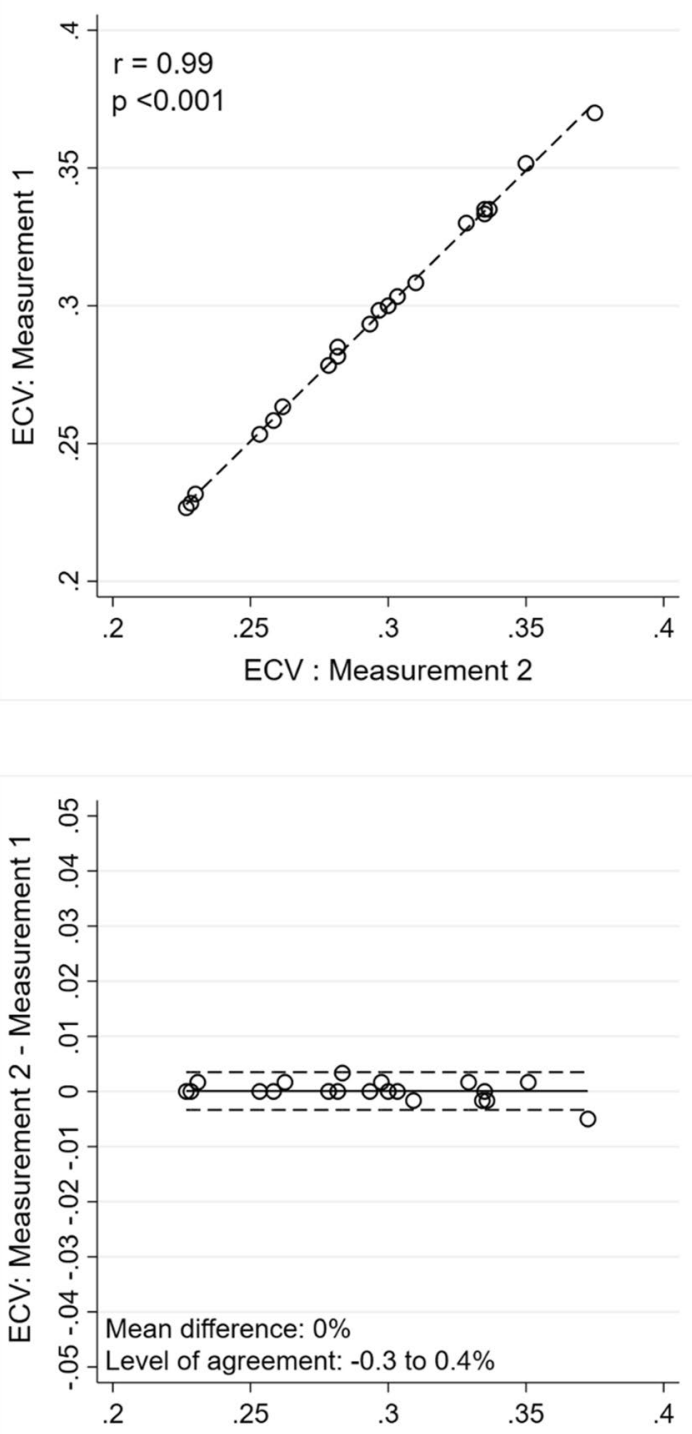

ECV: Mean of Measurement 1 and Measurement 2

Fig. 7 Intra-observer reproducibility of pre-contrast T1 relaxation time (left hand panels) and extracellular volume (right hand panels) measurements

Several clinical and pathological studies have revealed an association between bileaflet MVP with MAD, focal fibrosis within the infero-lateral LV segment or papillary muscles, and an increased risk of arrhythmia $[6,7,10,21$, 26]. Interestingly, in our cohort only $47 \%$ of MVP-MAD patients had LGE detected in the LV but $87 \%$ had a mean ECV above the upper limit of $27 \%$ in the basal LV myocardium. An ECVsyn $>27 \%$ was not only found in $93 \%$ of LGE-positive, but also in 81\% of LGE-negative MVP-MD patients. Moreover, an ECVsyn was increased in all the basal segments, suggesting that fibrotic remodelling is not restricted to the basal inferior/infero-lateral region, but develops in all myocardium adjacent to the insertion of the prolapsing valve. Our findings indicate that detection of confluent fibrosis using LGE alone may underestimate the fibrotic remodelling of the basal myocardium in relation to severe MVP and MAD.

The association between MVP and interstitial fibrosis, and its relation to VA has seldom been investigated. In a series published by Pradella et al. patients with MVP had 
Pre-contrast T1 relaxation
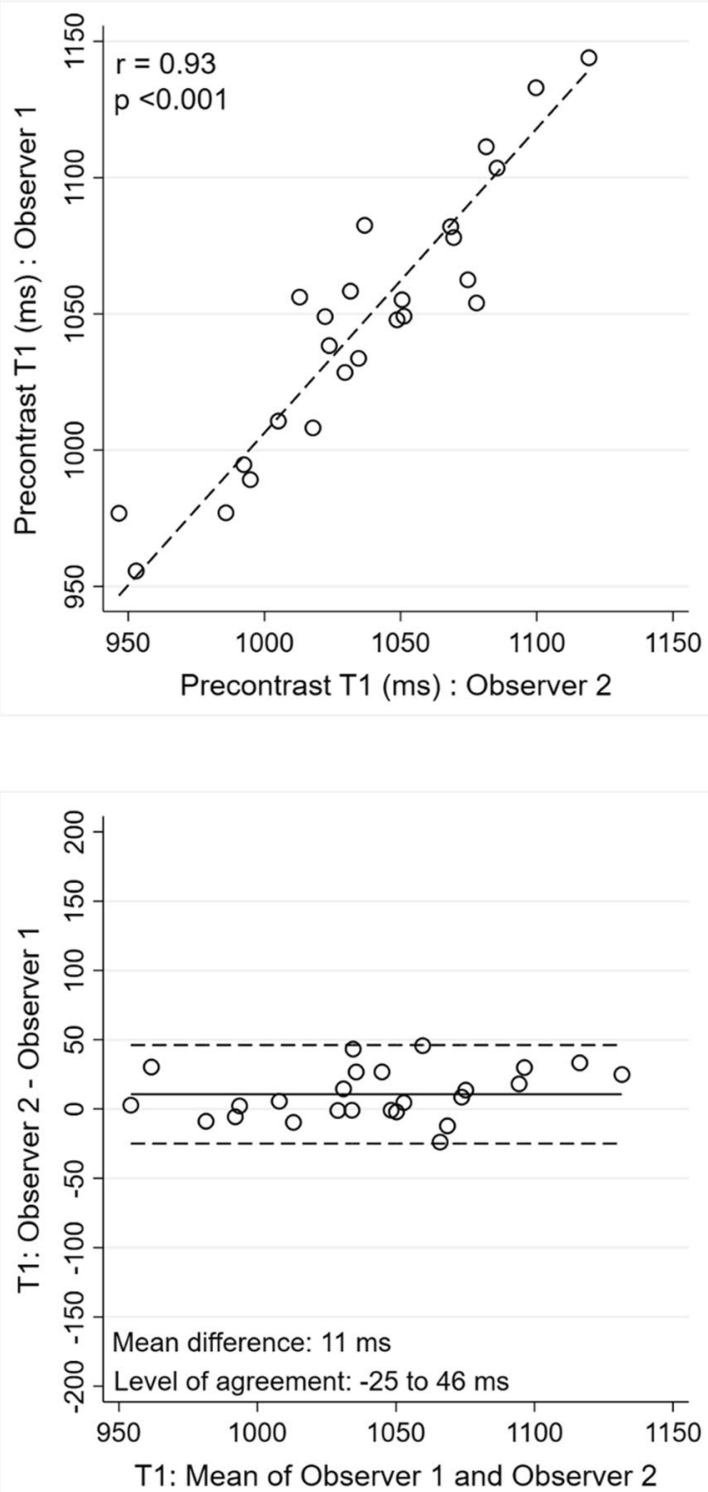

Extracellular volume
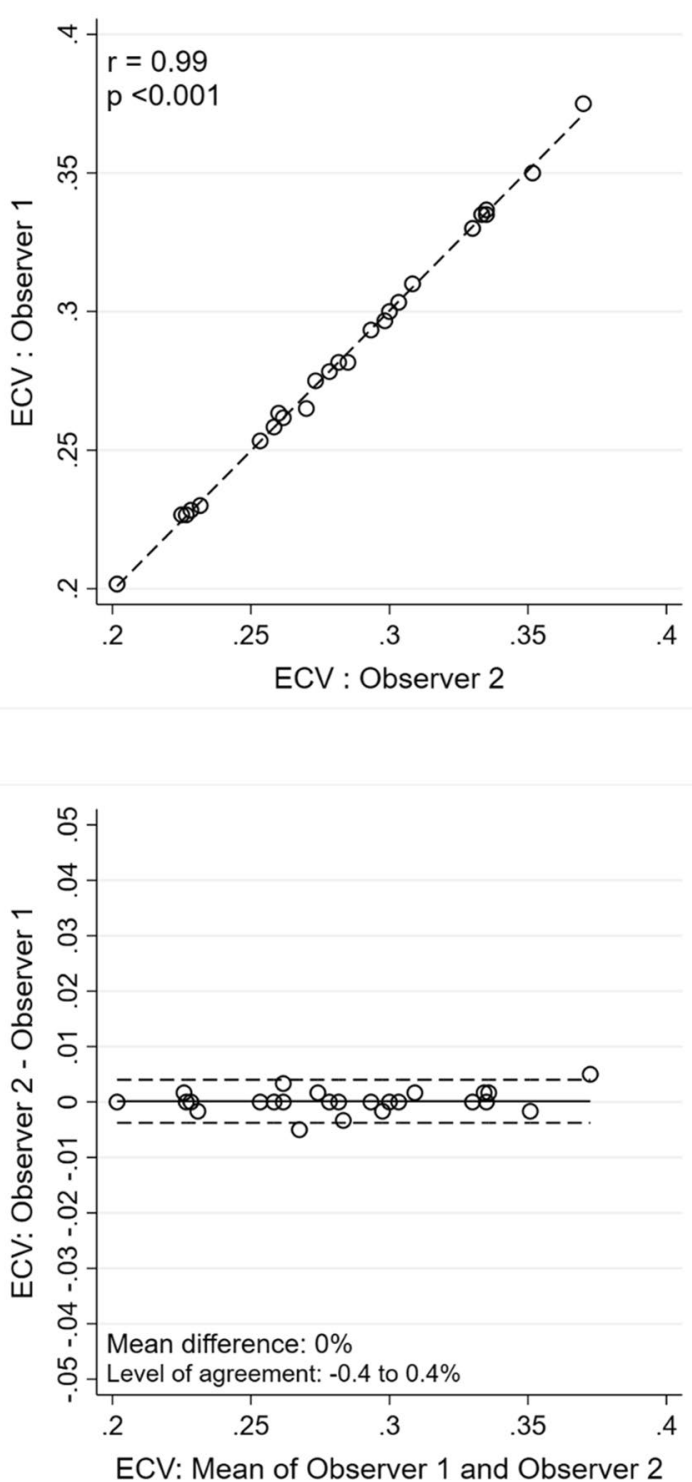

Fig. 8 Inter-observer reproducibility of pre-contrast T1 relaxation time (left hand panels) and extracellular volume (right hand panels) measurements

higher native and lower post-contrast T1 myocardial values, but no association with complex VA was found [17]. Bui et al. also detected an association between MVP and reduced post-contrast $\mathrm{T} 1$ times, suggesting increased myocardial fibrosis. Interestingly, in their series, MVP patients with complex VA had lower post-contrast T1 times compared to MVP patients with no arrhythmia making the authors conclude that interstitial remodelling may contribute to ventricular arrhythmogenesis [18].
However both studies did not evaluate the presence of MAD nor calculated the ECV. In the present study, the magnitude of the MAD was strongly correlated to the extent of interstitial fibrosis assessed by ECV, but was not associated with macroscopic fibrosis evaluated by LGE. This suggests that ECV may provide a more comprehensive assessment of LV remodelling induced by MVPMAD. Based on studies focusing on ischemic heart disease [32-34, 39], we hypothesized that the presence 
of interstitial fibrosis (i.e. high ECVsyn) could be predictive of VA in MVP-MAD patients. Rhythm assessment was obtained in 15 higher risk patients referred for suspected MVP arrhythmic syndrome and complex VA (either complex PVC or NSVT) were detected in almost all of them. Surprisingly, LGE was detected in only half of the patients but an increase in ECVsyn above the cutoff value was measured in all. Furthermore, ECVsyn was comparably increased in patients with and without LGE, supporting the hypothesis of an implication of interstitial fibrosis, and not only replacement fibrosis, in the development of VA in MVP-MAD patients. Several patterns of remodeling may be linked to the arrhythmic risk in MVP: either focal replacement fibrosis assessed by LGE or interstitial fibrosis measured with T1 mapping. This is in line with the description that VA in MVP can arise from the fascicular region or the septal regions of the mitral annulus in electrophysiological studies, and not only from regions presenting focal macroscopic fibrosis [4]. Finally and most importantly, we found a similar predictive value for OHCA between ECVsyn and LGE extent in our series, which supports systematically performing ECVsyn measurements in addition to LGE assessment during CMR examinations of patients with MVP. This parameter may provide additional prognostic value beyond LGE, as its fair specificity may complement the assessment of LGE in the unmet challenge of primary prevention risk stratification.

Given the retrospective nature of the study, synthetic haematocrit was used to calculate the ECV, as previously described in literature [24, 40,41]. Until now, some concerns have been raised regarding the reliability of synthetic haematocrit estimations, for instance in the presence of haematologic disorders [42] or in the paediatric population [43], where significant variations between measured and ECVsyn have been observed. Furthermore, the importance of a local calibration of the synthetic haematocrit, which can vary among different scanners, has been highlighted. In our center ECVsyn calculation has been calibrated from a large cohort of patients, no haematologic disorders were present and all patients were $>18$ years old. Therefore, the potential inaccuracies associated with this simplified method have been minimized and we believe that the ECVsyn used in this study reflects the true myocardial ECV of the population.

\section{Limitations}

The present study has several limitations. First, it is an observational, retrospective study, including a limited number of patients. Although our population was comparable in terms of arrhythmic MVP. Complex arrhythmia were detected in almost all of them, an evidence of a likely selection bias toward higher arrhythmic risk.
Fourth, although we used high resolution bright-blood LGE-CMR and images were interpreted by expert operators, routine evaluation of the presence of fibrosis in the papillary muscles area could prove challenging in routine and was not part of our analysis. Fifth, MR severity was not systematically evaluated during CMR examinations and we cannot assess the influence of MR severity on risk prediction. Sixth, no histologic confirmation of interstitial or replacement fibrosis could be obtained and all our results are based on indirect CMR markers of myocardial fibrosis. Finally, patients underwent to CMR examination in two different scanners (Siemens Aera and Siemens Sola); however local calibration of synthetic haematocrit was performed for both scanners, aim at to obtaining a reliable estimation of the synthetic haematocrit and ECVsyn.

\section{Conclusion}

In patients with MVP-MAD, remodelling of the LV occurs and both focal replacement and interstitial myocardial fibrosis can be detected by CMR. Compared to LGE extent, ECVsyn of the basal LV segments had a stronger association with MAD severity, and a similar association with OHCA. Our data suggest that ECVsyn should be part of the CMR examination of MVP patients in an effort to better assess fibrous remodelling as it may provide additional value beyond the assessment of LGE in the arrhythmic risk stratification.

\section{Abbreviations}

bSSFP: Balanced steady state free precession; CMR: Cardiovascular magnetic resonance; ECG: Electrocardiogram; ECVsyn: Synthetic extracellular volume; LA: Left atrium/left atrial; LGE: Late gadolinium enhancement; LV: Left ventricle/left ventricular; LVEDV: Left ventricular end-diastolic volume; LVEF: Left ventricular ejection fraction; LVESV: Left ventricular end-systolic volume; MAD: Mitral annular disjunction; MOLLI: Modified Look-Locker inversion recovery; MR: Mitral regurgitation; MVP: Mitral valve prolapse; NoMAD: No mitral annular disjunction; NSVT: Non-sustained ventricular tachycardia; OHCA: Out-of-hospital cardiac arrest; PVC: Premature ventricular contraction; SCD: Sudden cardiac death; VA: Ventricular arrhythmia.

\section{Supplementary Information}

The online version contains supplementary material available at https://doi. org/10.1186/s12968-021-00797-2.

Additional file 1: Figure S1. Relationship between haematocrit (\%) and R1 blood pool $\left(\mathrm{msec}^{-1}\right)$ in the two scanners used in the study (MAGNETOM Aera or Sola, Siemens Healthineers, Erlangen-Germany).

\section{Acknowledgements}

The authors thank all the technical staff at the Department of Radiology, in particular David Rodrigues Saraiva and Chantal Rohner for their skillful assistance.

\section{Authors' contributions}

AGP: contribute in conceiving the study, performed the CMR, collect the data, draft the manuscript and she is responsible for the overall content as 
guarantor; DA: collect the data and contribute in writing the manuscript; PP, SH, TR, APP: contribute in collecting data MLB, OM, EP, JS: critically revised the manuscript; PM: conceive the study, performed the CMR, draft the manuscript and he's responsible for the overall content as guarantor. All authors read and approved the final manuscript.

\section{Funding}

This clinical study was conducted without financial support.

\section{Availability of data and materials}

The datasets used and analysed during the current study are available from the corresponding author on reasonable request.

\section{Declarations}

\section{Ethics approval and consent to participate}

The CMR registry was approved by the local ethics committee CER-VD (Protocol number 2018-00656) and the patients provided written informed consent for their inclusion.

\section{Consent for publication}

Authors consent for publication of the manuscript.

\section{Competing interests}

Beyond the study's interests, Dr. Le Bloa received a training scholarship from the SIPCA Foundation, Prilly, Switzerland. Prof Schwitter received research grants from Bayer Healthcare, Switzerland. Other authors have nothing to disclose.

\section{Author details}

1 Department of Cardiology, Lausanne University Hospital (CHUV), Rue du Bugnon 46, 1011 Lausanne, Switzerland. ${ }^{2}$ Center for Cardiac Magnetic Resonance of the CHUV (CRMC), Lausanne University Hospital, Lausanne, Switzerland. ${ }^{3}$ University of Lausanne (UniL), Lausanne, Switzerland. ${ }^{4}$ Division of Cardiology, Fondazione Cardiocentro Ticino, Via Tesserete 48, CH-6900 Lugano, Switzerland.

Received: 2 March 2021 Accepted: 20 July 2021

Published online: 13 September 2021

\section{References}

1. Nordhues BD, Siontis KC, Scott CG, et al. Bileaflet mitral valve prolapse and risk of ventricular dysrhythmias and death. J Cardiovasc Electrophysiol. 2016;27:463-8.

2. Narayanan K, Uy-Evanado A, Teodorescu C, et al. Mitral valve prolapse and sudden cardiac arrest in the community. Heart Rhythm. 2016;13:498-503.

3. Freed LA, Levy D, Levine RA, et al. Prevalence and clinical outcome of mitral-valve prolapse. N Engl J Med. 1999;341:1-7.

4. Sriram CS, Syed FF, Ferguson ME, et al. Malignant bileaflet mitral valve prolapse syndrome in patients with otherwise idiopathic out-of-hospital cardiac arrest. J Am Coll Cardiol. 2013;62:222-30.

5. PerazzoloMarra M, Basso C, De Lazzari M, et al. Morphofunctional abnormalities of mitral annulus and arrhythmic mitral valve prolapse. Circ Cardiovasc Imaging. 2016;9:e005030.

6. Lancellotti P, Garbi M. Malignant mitral valve prolapse: substrates to ventricular remodeling and arrhythmias. Circ Cardiovasc Imaging. 2016;9:e005248

7. Dollar AL, Roberts WC. Morphologic comparison of patients with mitral valve prolapse who died suddenly with patients who died from severe valvular dysfunction or other conditions. J Am Coll Cardiol. 1991;17:921-31.

8. Essayagh B, Sabbag A, Antoine C, et al. Presentation and outcome of arrhythmic mitral valve prolapse. J Am Coll Cardiol. 2020;76:637-49.

9. Nishimura RA, McGoon MD, Shub C, Miller FA Jr, Ilstrup DM, Tajik AJ. Echocardiographically documented mitral-valve prolapse. Long-term follow-up of 237 patients. N Engl J Med. 1985;313:1305-9.

10. Basso C, Perazzolo Marra M, Rizzo S, et al. Arrhythmic mitral valve prolapse and sudden cardiac death. Circulation. 2015;132:556-66.
11. Dejgaard LA, Skjolsvik ET, Lie OH, et al. The mitral annulus disjunction arrhythmic syndrome. J Am Coll Cardiol. 2018;72:1600-9.

12. Lee $A P$, Jin $C N$, Fan $Y$, Wong RHL, Underwood MJ, Wan S. Functional implication of mitral annular disjunction in mitral valve prolapse: a quantitative dynamic 3D echocardiographic study. JACC Cardiovasc Imaging. 2017;10:1424-33.

13. Fontana M, White SK, Banypersad SM, et al. Comparison of T1 mapping techniques for ECV quantification. Histological validation and reproducibility of ShMOLLI versus multibreath-hold T1 quantification equilibrium contrast CMR. J Cardiovasc Magn Reson. 2012;14:88.

14. Taylor AJ, Salerno M, Dharmakumar R, Jerosch-Herold M. T1 mapping: basic techniques and clinical applications. JACC Cardiovasc Imaging. 2016:9:67-81.

15. Bing R, Cavalcante JL, Everett RJ, Clavel MA, Newby DE, Dweck MR. Imaging and impact of myocardial fibrosis in aortic stenosis. JACC Cardiovasc Imaging. 2019;12:283-96

16. Lee H, Park JB, Yoon YE, et al. Noncontrast myocardial T1 mapping by cardiac magnetic resonance predicts outcome in patients with aortic stenosis. JACC Cardiovasc Imaging. 2018;11:974-83.

17. Pradella S, Grazzini G, Brandani M, et al. Cardiac magnetic resonance in patients with mitral valve prolapse: focus on late gadolinium enhancement and T1 mapping. Eur Radiol. 2019;29:1546-54.

18. Bui AH, Roujol S, Foppa M, et al. Diffuse myocardial fibrosis in patients with mitral valve prolapse and ventricular arrhythmia. Heart. 2017:103:204-9.

19. Kitkungvan D, Yang EY, El Tallawi KC, et al. Extracellular volume in primary mitral regurgitation. JACC Cardiovasc Imaging. 2020;14:1146-60.

20. Guglielmo M, Fusini L, Muscogiuri G, et al. T1 mapping and cardiac magnetic resonance feature tracking in mitral valve prolapse. Eur Radiol. 2021;31:1100-9.

21. Han Y, Peters DC, Salton CJ, et al. Cardiovascular magnetic resonance characterization of mitral valve prolapse. JACC Cardiovasc Imaging. 2008;1:294-303.

22. Cerqueira MD, Weissman NJ, Dilsizian V, et al. Standardized myocardial segmentation and nomenclature for tomographic imaging of the heart. A statement for healthcare professionals from the Cardiac Imaging Committee of the Council on Clinical Cardiology of the American Heart Association. Circulation. 2002:105:539-42.

23. Vincenti G, Masci PG, Rutz T, et al. Impact of bileaflet mitral valve prolapse on quantification of mitral regurgitation with cardiac magnetic resonance: a single-center study. J Cardiovasc Magn Reson. 2017;19:56.

24. Treibel TA, Fontana M, Maestrini $V$, et al. Automatic measurement of the myocardial interstitium: synthetic extracellular volume quantification without haematocrit sampling. JACC Cardiovasc Imaging. 2016;9:54-63.

25. Kellman $\mathrm{P}$, Wilson $\mathrm{JR}$, Xue $\mathrm{H}$, Ugander $\mathrm{M}$, Arai AE. Extracellular volume fraction mapping in the myocardium, part 1: evaluation of an automated method. J Cardiovasc Magn Reson. 2012;14:63.

26. Boudoulas H, Schaal SF, Stang JM, Fontana ME, Kolibash AJ, Wooley CF. Mitral valve prolapse: cardiac arrest with long-term survival. Int J Cardiol. 1990;26:37-44

27. Chan RH, Maron BJ, Olivotto I, et al. Prognostic value of quantitative contrast-enhanced cardiovascular magnetic resonance for the evaluation of sudden death risk in patients with hypertrophic cardiomyopathy. Circulation. 2014;130:484-95.

28. Gao P, Yee R, Gula L, et al. Prediction of arrhythmic events in ischemic and dilated cardiomyopathy patients referred for implantable cardiac defibrillator: evaluation of multiple scar quantification measures for late gadolinium enhancement magnetic resonance imaging. Circ Cardiovasc Imaging. 2012;5:448-56.

29. Greulich S, Deluigi CC, Gloekler S, et al. CMR imaging predicts death and other adverse events in suspected cardiac sarcoidosis. JACC Cardiovasc Imaging. 2013;6:501-11.

30. Gulati A, Jabbour A, Ismail TF, et al. Association of fibrosis with mortality and sudden cardiac death in patients with nonischemic dilated cardiomyopathy. JAMA. 2013;309:896-908.

31. Flett AS, Hasleton J, Cook C, et al. Evaluation of techniques for the quantification of myocardial scar of differing etiology using cardiac magnetic resonance. JACC Cardiovasc Imaging. 2011;4:150-6.

32. Acosta J, Fernandez-Armenta J, Borras R, et al. Scar characterization to predict life-threatening arrhythmic events and sudden cardiac death in 
patients with cardiac resynchronization therapy: the GAUDI-CRT study. JACC Cardiovasc Imaging. 2018;11:561-72.

33. de Bakker JM, van Capelle FJ, Janse MJ, et al. Slow conduction in the infarcted human heart. "Zigzag" course of activation. Circulation. 1993;88:915-26.

34. Gardner PI, Ursell PC, Fenoglio JJ Jr, Wit AL. Electrophysiologic and anatomic basis for fractionated electrograms recorded from healed myocardial infarcts. Circulation. 1985;72:596-611.

35. Miller CA, Naish JH, Bishop P, et al. Comprehensive validation of cardiovascular magnetic resonance techniques for the assessment of myocardial extracellular volume. Circ Cardiovasc Imaging. 2013;6:373-83.

36. Moon JC, Messroghli DR, Kellman P, et al. Myocardial T1 mapping and extracellular volume quantification: a Society for Cardiovascular Magnetic Resonance (SCMR) and CMR Working Group of the European Society of Cardiology consensus statement. J Cardiovasc Magn Reson. 2013;15:92.

37. Treibel TA, Fridman $Y$, Bering $P$, et al. Extracellular volume associates with outcomes more strongly than native or post-contrast myocardial T1. JACC Cardiovasc Imaging. 2020;13:44-54.

38. Kitkungvan D, Nabi F, Kim RJ, et al. Myocardial fibrosis in patients with primary mitral regurgitation with and without prolapse. J Am Coll Cardiol. 2018;72:823-34.
39. Arbustini E, Kramer CM, Narula J. Arrhythmogenic potential of border zone after myocardial infarction: scar is more than just a healed wound. JACC Cardiovasc Imaging. 2018;11:573-6.

40. Fent GJ, Garg P, Foley JRJ, et al. Synthetic myocardial extracellular volume fraction. JACC Cardiovasc Imaging. 2017;10:1402-4.

41. Kammerlander AA, Duca F, Binder C, et al. Extracellular volume quantification by cardiac magnetic resonance imaging without haematocrit sampling: ready for prime time? Wien Klin Wochenschr. 2018;130:190-6.

42. Censi S, Cimaglia P, Barbieri A, et al. Performance of synthetic extracellular volume fraction in different cardiac phenotypes from a prospective cohort of patients referred for cardiac magnetic resonance. J Magn Reson Imaging. 2021;54:429-39.

43. Raucci FJ Jr, Parra DA, Christensen JT, et al. Synthetic haematocrit derived from the longitudinal relaxation of blood can lead to clinically significant errors in measurement of extracellular volume fraction in pediatric and young adult patients. J Cardiovasc Magn Reson. 2017;19:58.

\section{Publisher's Note}

Springer Nature remains neutral with regard to jurisdictional claims in published maps and institutional affiliations.
Ready to submit your research? Choose BMC and benefit from:

- fast, convenient online submission

- thorough peer review by experienced researchers in your field

- rapid publication on acceptance

- support for research data, including large and complex data types

- gold Open Access which fosters wider collaboration and increased citations

- maximum visibility for your research: over $100 \mathrm{M}$ website views per year

At BMC, research is always in progress.

Learn more biomedcentral.com/submissions 